\title{
REDUCIBLE HYPERPLANE SECTIONS OF THREEFOLDS: TWO COMPONENTS OF SECTIONAL GENUS ZERO*
}

\author{
Antonio Lanteri AND Andrea Luigi Tironi
}

\begin{abstract}
By using adjunction theory, we describe the smooth complex projective threefolds admitting a simple normal crossing divisor of the form $A+B$ among their hyperplane sections, both components $A$ and $B$ being smooth surfaces with sectional genus 0 , and one of them being nef or at worst an exceptional divisor of the first reduction mapping.
\end{abstract}

\section{Introduction}

Projective manifolds with an irreducible hyperplane section being a special variety have been studied since longtime [BS]. However the corresponding study for a reducible hyperplane section consisting of a simple normal crossing divisor whose components are special varieties was started only recently by Chandler, Howard and Sommese [CHS]. Results from [CHS] seem to indicate that classification in this setting can be harder for varieties of low dimension than in higher dimensions. In particular, let $X \subset \boldsymbol{P}^{N}$ be a smooth projective variety, let $L$ be its hyperplane bundle and suppose that $|L|$ contains an element $A+B$ where $A, B$ are smooth irreducible divisors meeting transversally (simple smooth decomposition). If both $\left(A, L_{A}\right)$ and $\left(B, L_{B}\right)$ have sectional genus zero, then the structure of $(X, L)$ is known, as well as the description of $A$ and $B$, if $\operatorname{dim} X \geq 4$ ([CHS], [LT]). In this paper we address the classification problem in the same set-up, when $\operatorname{dim} X=3$.

We would like to mention that in the course of our study we received the preprint of [BCS], where a similar set up is considered. In particular [BCS, (5.3)] provides the list of all possible numerical invariants concerning $A, B$, and $A \cap B$ inside them, when both pairs $\left(A, L_{A}\right),\left(B, L_{B}\right)$ are rational scrolls meeting along a smooth curve of positive genus. The case when this curve is rational is not considered there, since, as the authors say, it fits into a general result formulated for any dimension in [CHS]. However, in our opinion, the three dimensional setting deserves to be further studied also in this case.

*2000 Mathematics Subject Classification. Primary 13J30, 14J26; Secondary 14C20, 14N30.

Keywords and phrases. 3-folds, line bundles (ample and spanned), adjunction theory, simple normal crossing divisors, sectional genus, special varieties.

Received January 16, 2004. 
Here, assuming that at least one of $A$ and $B$ is a nef divisor (seminef decomposition) we obtain a classification result, working in a slightly more general setting than that described above. In fact, the adjunction theoretic classification techniques we use allow us to assume that the line bundle $L$ is simply ample and spanned and not necessarily very ample. The result is as follows.

THEOREM 0.1. Let $L$ be an ample and spanned line bundle on a connected projective manifold $X$ of dimension three. Assume that there is a seminef simple smooth decomposition $A+B \in|L|$. If $g\left(A, L_{A}\right)=g\left(B, L_{B}\right)=0$ then $(X, L)$ and $A, B$, after renaming, are as in the following cases:

1. a scroll over $\boldsymbol{P}^{1}$, with $A$ being a $\boldsymbol{P}^{1}$-bundle over $\boldsymbol{P}^{1}$ and $B$ a fiber of the scroll projection;

2. a quadric fibration $q: X \rightarrow \boldsymbol{P}^{1}$, with both $A$ and $B$ being $\boldsymbol{P}^{1}$-bundles over $\boldsymbol{P}^{\mathbf{1}}$ via $q$;

3. $X=\boldsymbol{P}(\mathscr{V})$, where $\mathscr{V}$ is an ample and spanned vector bundle of rank 2 on $\boldsymbol{F}_{1}$ with Chern classes $c_{1}(\mathscr{V})=[3 \mathfrak{I}+b \mathfrak{\dagger}], c_{2}(\mathscr{V})=2 b-4$ for an integer $b \geq 5$, where $I$ and $\mathfrak{f}$ denote the $(-1)$-section and a fiber of $\boldsymbol{F}_{1}$, and $L$ is the tautological line bundle on $X$. Moreover, $A \cong \boldsymbol{F}_{1}$ is a section of the scroll projection $\pi, B=\boldsymbol{P}\left(\mathscr{V}_{\gamma}\right)$ where $\gamma$ is a smooth element of the linear system $|2 \mathfrak{l}+2 \mathfrak{f}|$ on $\boldsymbol{F}_{1}$, and there exists a non-splitting exact sequence $0 \rightarrow[B]_{A} \rightarrow$ $\left.\pi\right|_{A} ^{*} \mathscr{V} \rightarrow L_{A} \rightarrow 0$; furthermore $h=A \cap B$ is isomorphic to $\gamma$ on $A$ and is a section on $B$

4. $X=\boldsymbol{P}(\mathscr{V})$, where $\mathscr{V}$ is an ample and spanned vector bundle of rank 2 on $\boldsymbol{P}^{2}$ with Chern classes $c_{1}(\mathscr{V})=4, c_{2}(\mathscr{V})=5$, and $L$ is the tautological line bundle on $X$. Let $\pi: X \rightarrow \boldsymbol{P}^{2}$ be the scroll projection; then $A \cong \boldsymbol{F}_{1}$, $\pi_{\mid A}: A \rightarrow \boldsymbol{P}^{2}$ being the contraction of the $(-1)$-section of $A, B=\boldsymbol{P}\left(\mathscr{V}_{\gamma}\right) \cong$ $\boldsymbol{P}^{1} \times \boldsymbol{P}^{1}, \gamma$ being a smooth conic; furthermore $h=A \cap B$ is a section of $B$ with $h^{2}=0$ and on $A$ it is a smooth element of the linear system $|2 E+2 F|$, where $E$ and $F$ denote the $(-1)$-section and a fiber, respectively;

5. $\left(\boldsymbol{P}^{1} \times \boldsymbol{P}^{2}, \mathcal{O}_{\boldsymbol{P}^{1} \times \boldsymbol{P}^{2}}(1,2)\right)$, with $A \in\left|\mathcal{O}_{\boldsymbol{P}^{1} \times \boldsymbol{P}^{2}}(1,0)\right|$ and $B$ a general element of $\left|\mathcal{O}_{\boldsymbol{P}^{1} \times \boldsymbol{P}^{2}}(0,2)\right|$

6. $\left(\boldsymbol{P}^{1} \times \boldsymbol{P}^{1} \times \boldsymbol{P}^{1}, \mathcal{O}_{\boldsymbol{P}^{1} \times \boldsymbol{P}^{1} \times \boldsymbol{P}^{1}}(1,1,1)\right)$, with $A \in\left|\mathcal{O}_{\boldsymbol{P}^{1} \times \boldsymbol{P}^{1} \times \boldsymbol{P}^{1}}(1,0,0)\right|$ and $B \in$ $\left|\mathcal{O}_{\boldsymbol{P}^{1} \times \boldsymbol{P}^{1} \times \boldsymbol{P}^{1}}(0,1,1)\right|$, up to reordering the factors;

7. $\left(\boldsymbol{P}\left(T_{\boldsymbol{P}^{2}}\right), \xi_{T_{P^{2}}}\right)$, where $\xi_{T_{\boldsymbol{P}^{2}}}$ stands for the tautological line bundle, or equivalently, $X \in\left|\mathcal{O}_{\boldsymbol{P}^{2} \times \boldsymbol{P}^{2}}(1,1)_{X}\right|$ and $L=\mathcal{O}_{\boldsymbol{P}^{2} \times \boldsymbol{P}^{2}}(1,1)_{X}$ with $A \in\left|\mathcal{O}_{\boldsymbol{P}^{2} \times \boldsymbol{P}^{2}}(1,0)_{X}\right|$ and $B \in\left|\mathcal{O}_{\boldsymbol{P}^{2} \times \boldsymbol{P}^{2}}(0,1)_{X}\right|$

8. $K_{X}+2 L$ is nef and big and $(X, L)$ admits $\left(\boldsymbol{P}^{3}, \mathcal{O}_{\boldsymbol{P}^{3}}(2)\right)$ as first reduction, the reduction morphism $\rho: X \rightarrow \boldsymbol{P}^{3}$ being the blowing-up at $s \leq 1$ points: if $s=0$ then $A, B \in\left|\mathcal{O}_{\boldsymbol{P}^{3}}(1)\right|$, while if $s=1$, i.e. $\rho$ is the blowing-up at a point $p$, then, up to renaming, either $A$ is the proper transform of a quadric cone having vertex at $p$ and $B$ is the exceptional divisor, or $A$ is the proper transform of a plane through $p$ and $B$ that of a plane not containing $p$.

Actually we prove a little bit more, since the seminefness of the decomposition $A+B$ is only used to manage the case when both polarized manifolds 
$\left(A, L_{A}\right),\left(B, L_{B}\right)$ are scrolls. The remaining cases are settled by Theorems $3.3,4.1$ and 4.2 .

On the other hand, contrary to what is known in dimension 2 , we do not know concrete examples of polarized threefolds $(X, L)$ admitting a simple smooth decomposition $A+B \in|L|$ not satisfying the assumption in Theorem 0.1. In fact, all pairs $\left(A, L_{A}\right),\left(B, L_{B}\right)$ appearing in [BCS, (5.3)] do not satisfy this assumption; however the corresponding structure of $(X, L)$, if any, is completely unknown.

Here is a sketch of the proof. The assumption that $A+B$ is a seminef decomposition implies that the hinge curve $h=A \cap B$ is rational. Then we can rely on the adjunction theoretic structure of $(X, L)$, provided by [CHS] to obtain a precise description of the varieties at hand via a case-by-case analysis. In particular, in Section 2 we prove that if $(X, L)$ is a scroll over a smooth surface, then there are only three possibilities, namely cases 3,4 and 5 in Theorem 0.1 . Moreover, for each variety appearing in Theorem 0.1 we also list all sectional genus zero decompositions of the reducible elements of $|L|$.

This work was done in the framework of the National Research Project "Geometry on Algebraic Varieties", supported by the MIUR of the Italian Government (Cofin 2002).

\section{Background material}

We work over the field of complex numbers $\boldsymbol{C}$ and we use the standard notation from algebraic geometry. The tensor products of line bundles are denoted additively. The pull-back $l^{*} \mathscr{F}$ of a vector bundle $\mathscr{F}$ on a projective variety $X$ by an embedding $l: Y \rightarrow X$ is denoted by $\mathscr{F}_{Y}$. We denote by $K_{X}$ the canonical bundle of a smooth projective variety $X$. A polarized manifold is a pair $(X, L)$ consisting of a smooth projective variety $X$ and an ample line bundle $L$ on $X$. For polarized manifolds we will use the adjunction theoretic terminology of [BS]. In particular we say that $(X, L)$ is a scroll over a smooth projective variety to mean that it is a scroll in the adjunction theoretic sense. As a consequence, e.g., the smooth quadric surface $\left(\boldsymbol{Q}^{2}, \mathcal{O}_{\boldsymbol{Q}^{2}}(1)\right)=$ $\left(\boldsymbol{P}^{1} \times \boldsymbol{P}^{1}, \mathcal{O}_{\boldsymbol{P}^{1} \times \boldsymbol{P}^{1}}(1,1)\right)$ is not considered as a scroll over $\boldsymbol{P}^{1}$.

Let $(X, L)$ be a smooth complex projective threefold polarized by an ample and spanned line bundle. Suppose that $|L|$ contains a divisor $A+B$ where $A, B$ are irreducible smooth surfaces meeting transversally along a smooth curve. We say that $A+B$ is a smooth decomposition for $L$. Of course one can also consider smooth decompositions consisting of more than two components. Therefore, sometimes we use the expression simple smooth decomposition (ssd for short) to emphasize that there are just two components.

We are interested in ssd $A+B \in|L|$ such that

$$
g\left(A, L_{A}\right)=g\left(B, L_{B}\right)=0 .
$$

Under this assumption both pairs $\left(A, L_{A}\right),\left(B, L_{B}\right)$ are polarized surfaces of sec- 
tional genus zero, hence they belong to one of the following two classes [F1, (12.1) and (5.10)]:

$$
\begin{aligned}
\mathscr{A} & :=\left\{\left(\boldsymbol{P}^{2}, \mathcal{O}_{\boldsymbol{P}^{2}}(u)\right), u=1,2,\left(\boldsymbol{Q}^{2}, \mathcal{O}_{Q^{2}}(1)\right)\right\} \quad \text { and } \\
\mathscr{B} & :=\left\{\left(\boldsymbol{F}_{e},\left[C_{0}+b f\right]\right), e \geq 0\right\} .
\end{aligned}
$$

For the class $\mathscr{B}$ of rational scrolls we used standard symbols; of course the integer $b$ has to satisfy the ampleness condition $b>e[\mathrm{Ha}, \mathrm{p} .380]$. Let $h:=A \cap B$. We call $h$ the hinge curve; by assumption it is a smooth curve. Its genus is given by

$$
2 g(h)-2=\left(K_{X}+A+B\right) A B=\left(K_{X}+L\right) A B .
$$

We recall the following lemma.

Lemma 1.1 ([T, Lemma 1]). Let $(X, L)$ be a threefold polarized by an ample and spanned line bundle $L$. Let $A+B \in|L|$ be a ssd. Then $g(X, L)=g\left(A, L_{A}\right)+$ $g\left(B, L_{B}\right)+A B L-1$.

We need some structure results to describe smooth decompositions occurring for some standard varieties of adjunction theory.

Lemma 1.2. Let $(X, L)$ be a threefold polarized by an ample and spanned line bundle L. Assume that $(X, L)$ is a scroll over a smooth curve $C$ and let $A+B \in|L|$ be a ssd. Then, after renaming, $\left(A, L_{A}\right) \cong\left(\boldsymbol{P}^{2}, \mathcal{O}_{\boldsymbol{P}^{2}}(1)\right),\left(B, L_{B}\right)$ is a scroll over $C$ or $\left(\boldsymbol{Q}^{2}, \mathcal{O}_{\mathbf{Q}^{2}}(1)\right)$, and $g(X, L)=g\left(B, L_{B}\right)=g(C)$.

Proof. Let $\pi: X \rightarrow C$ be the scroll projection and let $F \cong \boldsymbol{P}^{2}$ be a general fiber of $\pi$. We have $[A]_{F}=\mathcal{O}_{\boldsymbol{P}^{2}}(a)$ and $[B]_{F}=\mathcal{O}_{\boldsymbol{P}^{2}}(b)$, for some integers $a, b \geq 0$. Since

$$
\mathcal{O}_{\boldsymbol{P}^{2}}(1)=L_{F}=[A]_{F}+[B]_{F}=\mathcal{O}_{\boldsymbol{P}^{2}}(a+b),
$$

it follows, after renaming, that $a=0, b=1$. This implies that $\left(A, L_{A}\right) \cong$ $\left(F, L_{F}\right) \cong\left(\boldsymbol{P}^{2}, \mathcal{O}_{\boldsymbol{P}^{2}}(1)\right)$ and $\left(B, L_{B}\right)$ is a scroll over $C$ or, possibly, $\left(\boldsymbol{Q}^{2}, \mathcal{O}_{\boldsymbol{Q}^{2}}(1)\right)$, if $g(C)=0$. Moreover

$$
A B L=[B]_{F}[L]_{F}=L_{F}^{2}=1
$$

and then by Lemma 1.1 we deduce that $g(X, L)=g\left(B, L_{B}\right)=g(C)$.

Lemma 1.3. Let $(X, L)$ be a Del Pezzo threefold polarized by an ample and spanned line bundle $L$. Assume that $A+B \in|L|$ is a ssd. Then $g\left(A, L_{A}\right)=$ $g\left(B, L_{B}\right)=0$ and $(X, L)$ is one of the following pairs:

1. $\left(\boldsymbol{P}^{1} \times \boldsymbol{P}^{1} \times \boldsymbol{P}^{1}, \mathcal{O}_{\boldsymbol{P}^{1} \times \boldsymbol{P}^{1} \times \boldsymbol{P}^{1}}(1,1,1)\right)$;

2. $\left(\boldsymbol{P}\left(T_{\boldsymbol{P}^{2}}\right), \xi_{T_{P^{2}}}\right)$, where $T_{\boldsymbol{P}^{2}}$ is the tangent bundle to $\boldsymbol{P}^{2}$ and $\xi_{T_{\boldsymbol{P}^{2}}}$ stands for the tautological line bundle;

3. $(X, L)$ has $\left(\boldsymbol{P}^{3}, \mathcal{O}_{\boldsymbol{P}^{3}}(2)\right)$ as first reduction, with $X$ being $\boldsymbol{P}^{3}$ blown-up at one point at most. 
Proof. By the genus formula we have that

$$
2 g(h)-2=\left(K_{X}+L\right) A B=-A B L<0,
$$

hence $A B L=2$. By Lemma 1.1 we thus get

$$
1=g(X, L)=g\left(A, L_{A}\right)+g\left(B, L_{B}\right)+1,
$$

which gives $g\left(A, L_{A}\right)=g\left(B, L_{B}\right)=0$. Then the assertion follows from [CHS, (3.4)].

Remarks. In case 1 of Lemma 1.3 we get a ssd $A+B \in|L|$, by choosing, e.g., $A \in\left|\mathcal{O}_{\boldsymbol{P}^{1} \times \boldsymbol{P}^{1} \times \boldsymbol{P}^{1}}(1,0,0)\right|$ and $B \in\left|\mathcal{O}_{\boldsymbol{P}^{1} \times \boldsymbol{P}^{1} \times \boldsymbol{P}^{1}}(0,1,1)\right|$. For this decomposition, $\left(A, L_{A}\right) \cong\left(\boldsymbol{Q}^{2}, \mathcal{O}_{\boldsymbol{Q}^{2}}(1)\right)$ and $\left(B, L_{B}\right) \in \mathscr{B}$.

In case $2, X$ can be described alternatively as a smooth element of $\left|\mathcal{O}_{\boldsymbol{P}^{2} \times \boldsymbol{P}^{2}}(1,1)\right|$ and $L=\mathcal{O}_{\boldsymbol{P}^{2} \times \boldsymbol{P}^{2}}(1,1)_{X}$. This shows that the only possible decomposition for $L$ is $A+B$, where $A \in\left|\mathcal{O}_{\boldsymbol{P}^{2} \times \boldsymbol{P}^{2}}(1,0)_{X}\right|$ and $B \in\left|\mathcal{O}_{\boldsymbol{P}^{2} \times \boldsymbol{P}^{2}}(0,1)_{X}\right|$, giving a scroll structure on both components.

As to 3, note that the case with $\rho: X \rightarrow \boldsymbol{P}^{3}$ the blowing-up at a point $p$ does really occur. Recall that $L:=\rho^{*}\left(\mathcal{O}_{\boldsymbol{P}^{3}}(2)\right)-E$, where $E$ is the exceptional divisor. Here we list all possible decompositions for $L$. Let $B^{\prime} \subset \boldsymbol{P}^{3}$ be an element of $\left|\mathcal{O}_{\boldsymbol{P}^{3}}(2)\right|$ containing $p$.

a) Suppose that $B^{\prime}$ is irreducible and let $B=\rho^{-1}\left(B^{\prime}\right)$ be its proper transform. If $B^{\prime}$ is smooth at $p$, then $B=\rho^{*} B^{\prime}-E \in|L|$ is an irreducible element. If $B^{\prime}$ is a quadric cone with vertex at $p$, then $B=\rho^{*} B^{\prime}-2 E \in|L-E|$. Moreover $B \cong \boldsymbol{F}_{2}$ with $\left(\rho^{*}\left(\mathcal{O}_{\boldsymbol{P}^{3}}(1)\right)\right)_{B}=\left[C_{0}+2 f\right]$, since $\rho: B \rightarrow B^{\prime}$ is the minimal desingularization. Here $C_{0}$ is a section of minimal self-intersection and $f$ is a fiber of $\boldsymbol{F}_{2}$. So, letting $A=E$, we get the ssd $A+B \in|L|$. Note that the curve $h=A \cap B$ is $C_{0}$ on $B$ and a conic on $A$. This gives

and

$$
L_{A}=[A]_{A}+[B]_{A}=\mathcal{O}_{\boldsymbol{P}^{2}}(-1+2)=\mathcal{O}_{\boldsymbol{P}^{2}}(1)
$$

$$
L_{B}=\left(2 \rho^{*}\left(\mathcal{O}_{\boldsymbol{P}^{3}}(1)\right)-E\right)_{B}=\left[C_{0}+4 f\right] .
$$

Therefore $\left(A, L_{A}\right) \cong\left(\boldsymbol{P}^{2}, \mathcal{O}_{\boldsymbol{P}^{2}}(1)\right),\left(B, L_{B}\right) \cong\left(\boldsymbol{F}_{2},\left[C_{0}+4 f\right]\right)$. $i=1,2$.

b) Suppose that $B^{\prime}=B_{1}^{\prime}+B_{2}^{\prime}$, where $B_{i}^{\prime} \in\left|\mathcal{O}_{\boldsymbol{P}^{3}}(1)\right|$, and let $B_{i}=\rho^{-1}\left(B_{i}^{\prime}\right)$,

b1) Let $p \in B_{1}^{\prime} \backslash B_{2}^{\prime}$. Then $B_{1}=\rho^{*}\left(B_{1}^{\prime}\right)-E \in\left|\rho^{*}\left(\mathcal{O}_{\boldsymbol{P}^{3}}(1)\right)-E\right|$; moreover $B_{1} \cong \boldsymbol{F}_{1}$ with $\left(\rho^{*}\left(\mathcal{O}_{\boldsymbol{P}^{3}}(1)\right)\right)_{B_{1}}=\left[C_{0}+f\right]$. Here $C_{0}$ is a section of minimal selfintersection and $f$ is a fiber of $\boldsymbol{F}_{1}$. Letting $A=B_{2}$ we get the ssd $A+B_{1} \in|L|$. Note that $h=A \cap B_{1}$ is a line inside $A$ and an element of $\left|C_{0}+f\right|$ as a curve on $B_{1}$. This gives

$$
L_{A}=[A]_{A}+\left[B_{1}\right]_{A}=\mathcal{O}_{P^{2}}(2)
$$

and

$$
L_{B_{1}}=\left(2 \rho^{*}\left(\mathcal{O}_{\boldsymbol{P}^{3}}(1)\right)-E\right)_{B_{1}}=\left[C_{0}+2 f\right],
$$

since $E \cap B_{1}$ is $C_{0}$. Therefore $\left(A, L_{A}\right) \cong\left(\boldsymbol{P}^{2}, \mathcal{O}_{\boldsymbol{P}^{2}}(1)\right),\left(B_{1}, L_{B_{1}}\right) \cong\left(\boldsymbol{F}_{1},\left[C_{0}+2 f\right]\right)$. 
b2) Let $p \in B_{1}^{\prime} \cap B_{2}^{\prime}$, but $B_{1}^{\prime} \neq B_{2}^{\prime}$. Then, arguing as before and letting $A=E$, we get the non simple smooth decomposition $A+B_{1}+B_{2} \in|L|$, where $\left(A, L_{A}\right) \cong\left(\boldsymbol{P}^{2}, \mathcal{O}_{\boldsymbol{P}^{2}}(1)\right)$ and $\left(B_{i}, L_{B_{i}}\right) \cong\left(\boldsymbol{F}_{1},\left[C_{0}+2 f\right]\right)$ for $i=1,2$.

b3) Finally let $B_{1}^{\prime}=B_{2}^{\prime}$. Then, letting $A=E$ we get the non-reduced decomposition $A+2 B_{1} \in|L|$, with $\left(A, L_{A}\right),\left(B_{1}, L_{B_{1}}\right)$ as in b2).

Note that the ssd found in a) and b1) correspond to the two cases mentioned in 8 of Theorem 0.1 .

Lemma 1.4. Let $(X, L)$ be a threefold polarized by an ample and spanned line bundle L. Assume that $(X, L)$ is a quadric fibration over a smooth curve $C$ and that $A+B \in|L|$ is a ssd. Then one of the following cases occurs:

1. $A$ is a fiber, up to renaming, and $g(X, L)=g\left(B, L_{B}\right)+1$;

2. $\left(A, L_{A}\right)$ and $\left(B, L_{B}\right)$ are scrolls over $C$ or $\left(\boldsymbol{Q}^{2}, \mathcal{O}_{Q^{2}}(1)\right)$, and $h$ is a section of both.

Moreover, under the assumption that $g\left(A, L_{A}\right)=g\left(B, L_{B}\right)=0$, only case 2 can occur.

Proof. Let $q: X \rightarrow C$ be the fibration and let $F \cong \boldsymbol{P}^{1} \times \boldsymbol{P}^{1}$ be a smooth fiber. Suppose that $A=F$; then

$$
L_{F}=[B]_{F}=\mathcal{O}_{\boldsymbol{P}^{1} \times \boldsymbol{P}^{1}}(1,1) .
$$

In particular, $A B L=\left(L_{F}\right)^{2}=2$. Moreover $K_{X}+2 L=q^{*} H \equiv t A$ for some integer $t \geq 1, H$ being an ample line bundle on $C$. Hence, by adjunction we get the following expressions:

$$
\begin{aligned}
& 2 g\left(A, L_{A}\right)-2=\left(K_{X}+L+A\right) A L=t A^{2} L-A B L=-A B L=-2, \\
& 2 g\left(B, L_{B}\right)-2=\left(K_{X}+L+B\right) B L=t A B L-A B L=(t-1) A B L=2(t-1) .
\end{aligned}
$$

Thus $g\left(A, L_{A}\right)=0, g\left(B, L_{B}\right)=t$, and then $g(X, L)=g\left(B, L_{B}\right)+1$, by Lemma 1.1.

Now suppose that neither $A$ nor $B$ are fibers. Then both $[A]$ and $[B]$ restrict non trivially to the general fiber $F$ and since $L_{F} \cong \mathcal{O}_{\boldsymbol{P}^{1} \times \boldsymbol{P}^{1}}(1,1)$, up to renaming, we see that $[A]_{F} \cong \mathcal{O}_{\boldsymbol{P}^{1} \times \boldsymbol{P}^{1}}(1,0), \quad[B]_{F} \cong \mathcal{O}_{\boldsymbol{P}^{1} \times \boldsymbol{P}^{1}}(0,1)$. Let $\Delta \subset C$ denote the image of the singular fibers of $q$ and set $U=\left.A \backslash q\right|_{A} ^{-1}(\Delta)$. Since the general fiber of $\left.q\right|_{A}$ is a $\boldsymbol{P}^{1}, A$ is a ruled surface over $C$. Set $\mathrm{e}=A \cap F$ and $\mathfrak{\mathfrak { f }}=B \cap F$. Note that $1=\mathfrak{e} \tilde{f}=A B F$. Hence, from the equality

$$
0=\mathrm{e}^{2}=\left(A_{F}\right)^{2}=[F]_{A}[A]_{A}=[F]_{A}\left(L_{A}-[B]_{A}\right)=[F]_{A} L_{A}-F A B=\mathrm{e} L_{A}-1,
$$

we see that $\mathfrak{e}$, the general fiber of $\left.q\right|_{A}$ is a line. Since $L_{A}$ is ample, this implies that every fiber of $\left.q\right|_{A}$ is a line. Thus $\left(A, L_{A}\right)$ is a scroll over $C$ or $\left(\boldsymbol{Q}^{2}, \mathcal{O}_{Q^{2}}(1)\right)$. Moreover, since $h \cap F=\mathfrak{e} \cap \mathfrak{f}$, we have that $\left.q\right|_{A}: h \rightarrow C$ is an isomorphism, i.e., $h$ is a section of $\left(A, L_{A}\right)$. Of course the same argument works for $\left(B, L_{B}\right)$.

This proves the first part of the statement. As to the last assertion, note that under our assumption on the sectional genera, in case 1 we would get $g(X, L)=1$. According to the classification of polarized manifolds this would imply that $(X, L)$ is not a quadric fibration $[\mathrm{F} 1,(12.3)]$, a contradiction. 
Example. Let $X=\boldsymbol{P}^{1} \times \boldsymbol{F}_{e} \quad$ (any $e \geq 0$ ) and $L=p_{1}^{*} \mathcal{O}_{\boldsymbol{P}^{1}}(1)+p_{2}^{*}[\mathrm{I}+b \mathfrak{f}]$ $(b>e)$, where $p_{1}, p_{2}$ are the two projections, and $\mathfrak{I}, \mathfrak{f}$ are the fundamental section and a fiber of $\boldsymbol{F}_{e}$ respectively. Note that $L$ is very ample. The composition of $p_{2}$ with the scroll projection of $\left(\boldsymbol{F}_{e},[\mathrm{I}+b \tilde{\mathfrak{f}}]\right)$ gives a morphism $q: X \rightarrow \boldsymbol{P}^{1}$ making $(X, L)$ a fibration with all fibers being smooth quadrics. Actually for any fiber $F$ of $q$ we have $F=\mathfrak{f} \times f$, where $\mathfrak{\mathfrak { T }}=p_{2}(F)$ and $f$ is a fiber of $p_{2}$, hence $F \cong$ $\boldsymbol{F}_{0}=\boldsymbol{P}^{1} \times \boldsymbol{P}^{1} . \quad$ For any curve $\Gamma \subset \boldsymbol{F}_{e}$ we set $\sigma_{\Gamma}=\sigma \cap p_{2}^{-1}(\Gamma)$, where $\sigma$ is a fiber of $p_{1}$, i.e., $\sigma \in\left|p_{1}^{*} \mathcal{O}_{\boldsymbol{P}^{1}}(1)\right|$. Thus $L_{F}=\left.p_{1}\right|_{F} ^{*} \mathcal{O}_{\boldsymbol{P}^{1}}(1)+\left.p_{2}\right|_{F} ^{*}[\mathbf{I}+b \mathfrak{\mathfrak { f }}]=\left[\sigma_{\mathfrak{f}}+f\right]$ is the sum of the two rulings, i.e., $\left(F, L_{F}\right)=\left(\boldsymbol{Q}^{2}, \mathcal{O}_{Q^{2}}(1)\right)$.

Let $A \in\left|p_{1}^{*} \mathcal{O}_{\boldsymbol{P}^{1}}(1)\right|$ and let $B \in\left|p_{2}^{*}[\mathrm{I}+b \mathfrak{f}]\right|$ be a smooth element (there is such a $B$ because $[\mathfrak{l}+b \mathfrak{f}]$ with $b>e$ is very ample). Then $A+B \in|L|$ is a ssd. Moreover, we have that

$$
\left(A, L_{A}\right) \cong\left(\boldsymbol{F}_{e},[\mathfrak{I}+b \mathfrak{f}]\right), \quad\left(B, L_{B}\right) \cong\left(\boldsymbol{P}^{1} \times \gamma=\boldsymbol{F}_{0},\left[\sigma_{\gamma}+\left(\gamma^{2}\right) f\right]\right),
$$

where $\gamma$ is a smooth curve in $|\mathfrak{l}+b \mathfrak{f}|$. Note that $\sigma_{\gamma} \cong \gamma$ via $p_{2}$. Moreover, $\gamma^{2}=2 b-e \geq 2$ and so $\left(B, L_{B}\right)$ is in class $\mathscr{B}$, i.e., it is not $\left(\boldsymbol{Q}^{2}, \mathcal{O}_{Q^{2}}(1)\right)$. Furthermore $h \cong \sigma_{\gamma}$, hence $g(h)=0$. Since

$$
A B L=A B(A+B)=A B^{2}=\gamma^{2}=2 b-e,
$$

recalling Lemma 1.1 we get $g(X, L)=2 b-e-1$. We would like to note also that in this case we can decompose an element of $|L|$ in at most $b+2$ smooth irreducible components, all of sectional genus zero, by taking $A$ as above and $B_{0} \in\left|p_{2}^{*}\right| \mathrm{l}, \quad B_{j} \in\left|p_{2}^{*} \mathfrak{f}\right|$ for every $j=1, \ldots, b$. Thus we obtain

$$
\begin{aligned}
&\left(A, L_{A}\right) \cong\left(\boldsymbol{F}_{e},[\mathfrak{I}+b \mathfrak{f}]\right), \quad\left(B_{0}, L_{B_{0}}\right) \cong\left(\boldsymbol{F}_{0},\left[\sigma_{\mathrm{l}}+(b-e) f\right]\right) \quad \text { and } \\
&\left(B_{j}, L_{B_{j}}\right) \cong\left(\boldsymbol{F}_{0},\left[\sigma_{\mathfrak{f}}+f\right]\right)
\end{aligned}
$$

for $j=1, \ldots, b$.

Finally, note that for $e=0$ we get $X=\boldsymbol{P}^{1} \times \boldsymbol{P}^{1} \times \boldsymbol{P}^{1}$ with $L=$ $\mathcal{O}_{\boldsymbol{P}^{1} \times \boldsymbol{P}^{1} \times \boldsymbol{P}^{1}}(1,1, b), b \geq 1$. For $b=1$ our $(X, L)$ is a Del Pezzo threefold, while, for $b \geq 2$ we get a quadric fibration and $g(X, L)=2 b-1$.

\section{Scrolls over surfaces}

In this Section we classify

(2.0) threefolds $X$ endowed with an ample and spanned line bundle $L$ such that $(X, L)$ is a scroll over a smooth surface $S$, and there is a ssd $A+B \in|L|$ with $g\left(A, L_{A}\right)=g\left(B, L_{B}\right)=0$.

Recall that by scroll we mean an adjunction-theoretic scroll. However, by [S, Theorem 3.3 (note that the proof there works also for $k=1$ )], our $(X, L)$ is also a classical scroll over $S$. Let $\pi: X \rightarrow S$ be the scroll projection; since $L$ restricts to every fiber as $\mathcal{O}_{P^{1}}(1)$, up to renaming, we have that $\left.\pi\right|_{A}: A \rightarrow S$ is a birational morphism, while $B=\pi^{*} \gamma$, where $\gamma \subset S$ is a smooth curve. Moreover, since $g\left(A, L_{A}\right)=g\left(B, L_{B}\right)=0$ we have that $\left(A, L_{A}\right)$ as well as $\left(B, L_{B}\right)$ are among the pairs listed in (1.0). In particular this implies that $\gamma \cong \boldsymbol{P}^{1}$ and $S$ is either 
$\boldsymbol{P}^{2}$ or $\boldsymbol{F}_{e}$ for some $e \geq 0$, being dominated birationally by $A$. Moreover there are two possibilities: either

i) $\left.\pi\right|_{A}$ is an isomorphism, or

ii) $\left.\pi\right|_{A}$ contracts some $(-1)$-line.

Since $(X, L)$ is a scroll over $S$, we can write $X=\boldsymbol{P}(\mathscr{V})$ where $\mathscr{V}:=\pi_{*} L$ is an ample and spanned vector bundle of rank 2 on $S$. Moreover, since $L$ is the tautological line bundle of $\mathscr{V}$, we know that $K_{X}+2 L=\pi^{*} H$, where $H=$ $K_{S}+\operatorname{det} \mathscr{V}$ is an ample line bundle on $S$.

By the genus formula we have both

$$
-2=2 g\left(A, L_{A}\right)-2=\left(K_{X}+A+L\right) L A=\left(K_{X}+2 L-B\right) A L=\pi^{*} H A L-A B L
$$

and

$$
-2=2 g\left(B, L_{B}\right)-2=\left(K_{X}+B+L\right) L B=\left(K_{X}+2 L-A\right) B L=\pi^{*} H B L-A B L .
$$

The two genera being equal, this gives

$$
\pi^{*} H A L=\pi^{*} H B L=\pi^{*} H \pi^{*} \gamma L=H \gamma .
$$

Moreover

$$
H \operatorname{det} \mathscr{V}=\pi^{*} H L^{2}=\pi^{*} H L(A+B)=2 \pi^{*} H B L=2 H \gamma .
$$

Theorem 2.1. Let $X, L, A, B$ be as in (2.0) and let $S \neq \boldsymbol{P}^{2}$. Then $X=\boldsymbol{P}(\mathscr{V})$, where $\mathscr{V}$ is an ample and spanned vector bundle of rank 2 on $\boldsymbol{F}_{1}$ with Chern classes $c_{1}(\mathscr{V})=[3 \mathfrak{I}+b \mathfrak{\uparrow}], c_{2}(\mathscr{V})=2 b-4$ for an integer $b \geq 5$, where $\mathbb{I}$ and $\mathfrak{f}$ denote the (-1)-section and a fiber of $\boldsymbol{F}_{1}$, and $L$ is the tautological line bundle on $X$. Moreover $A \cong \boldsymbol{F}_{1}$ is a section of $X, B=\boldsymbol{P}\left(\mathscr{V}_{\gamma}\right)$, where $\gamma$ is a smooth element of the linear system $|2 \mathfrak{I}+2 \mathfrak{f}|$, and the scroll projection $\pi$ induces a non-splitting exact sequence $\left.0 \rightarrow[B]_{A} \rightarrow \pi\right|_{A} ^{*} \mathscr{V} \rightarrow L_{A} \rightarrow 0$; furthermore $h=A \cap B$ is isomorphic to $\gamma$ on $A$ and is a section on $B$.

Proof. By what we said before, $S=\boldsymbol{F}_{e}$ for some $e \geq 0$. Moreover, in view of (1.0), we are in case i). Letting $X=\boldsymbol{P}(\mathscr{V})$, where $\mathscr{V}=\pi_{*} L$ as before, now we can write det $\mathscr{V}=[a \mathbf{l}+b \mathfrak{f}]$ for some suitable integers $a$ and $b$. Moreover $K_{X}+2 L=\pi^{*} H$, where

$$
H=K_{\boldsymbol{F}_{e}}+\operatorname{det} \mathscr{V}=[(a-2) \mathfrak{l}+(b-2-e) \tilde{\mathfrak{f}}],
$$

is an ample line bundle, hence

$$
a>2 \text { and } b>(a-1) e+2,
$$

in view of [Ha, p. 380]. Since $\left(A, L_{A}\right)$ is either $\left(\boldsymbol{Q}^{2}, \mathcal{O}_{\boldsymbol{Q}^{2}}(1)\right)$ or a scroll by (1.0), we can identify $A$ with $\boldsymbol{F}_{e}$ via the isomorphism $\left.\pi\right|_{A}$ and write (up to exchanging the factors in case $e=0) L_{A}=[\mathrm{I}+t \tilde{\mathrm{\eta}}]$ for some integer $t>e$. Thus, in view of the identification

$$
h=A \cap B=A \cap \pi^{*} \gamma=\gamma
$$


induced by $\left.\pi\right|_{A}$, we get

$$
A B L=h L_{A}=\gamma(\mathfrak{I}+t \mathfrak{\Upsilon}) .
$$

Moreover, since $\pi^{*} H A L=\left(\left.\pi\right|_{A} ^{*} H\right) L_{A}$, (2.1) gives

$$
H(\mathrm{I}+t \mathfrak{\uparrow})=H \gamma .
$$

Now, as already observed, $B=\pi^{*} \gamma$, where $\gamma \subset \boldsymbol{F}_{e}$ is a smooth rational curve. Hence, by [Ha, p. 380] an easy check leads to the following possibilities:

(1) $\gamma=\mathfrak{l}$,

(2) $\gamma=\mathfrak{f}$,

(3) $\gamma \sim \mathfrak{l}+m \mathfrak{f}$, for some integer $m \geq e$,

(4) $e=0$ and $\gamma \sim m \mathfrak{l}+\mathfrak{f}$, for some integer $m \geq 2$,

(5) $e=1$ and $\gamma \sim 2 \mathfrak{l}+2 \mathfrak{f}$.

We proceed with a case-by-case analysis.

Case (1). We have $H \gamma=H \mathrm{l}$, but this contradicts (2.5).

Case (2). We have $H \gamma=H \mathfrak{\uparrow}$. But (2.5) says that $H \gamma=H \mathfrak{\uparrow}+H(\mathfrak{l}+$ $(t-1) \mathfrak{f})>H \mathfrak{f}$, a contradiction.

Case (3). We have $H \gamma=(a-2)(m-e)+b-e-2$. Moreover (2.5) immediately gives $m=t$ and then $A B L=2 m-e$, by (2.4). By the condition $g\left(B, L_{B}\right)=0$, recalling also $(2.1)$ we thus get $b=(4-a) m+(a-2) e$. Combining this with (2.3) we have $(4-a) m>e+2>0$ and so, recalling (2.3) we conclude that $a=3$. Hence $b=m+e$. Replacing these values into the expressions of $H \operatorname{det} \mathscr{V}$ and $H \gamma,(2.2)$ gives a numerical contradiction.

Case (4). In this case we have $H \operatorname{det} \mathscr{V}=2(a b-a-b)$ and $H \gamma=a-2+$ $m(b-2)$. So, noting that $b>2$ by (2.3), (2.2) gives $m=a-1$. But (2.5) in turn shows that $t=b-3$. Thus $H \gamma=m t+2 m-1$. On the other hand (2.4) shows that $A B L=m t+1$. So, recalling also (2.1), the condition $g\left(B, L_{B}\right)=0$ leads to the equality $m=0$, which is a contradiction.

Thus we are in Case (5). In particular, $S=\boldsymbol{F}_{1}$ and $H=[(a-2) \mathfrak{I}+$ $(b-3) \tilde{\mathfrak{f}}$. We have $H \operatorname{det} \mathscr{V}=(a-2)(b-a)+a(b-3)$ and $2 H \gamma=4(b-3)$. So (2.2) gives $(a-2)(b-a)=(4-a)(b-3)$. Recalling (2.3) we deduce that $2 \leq b-a \leq(4-a)(b-3)$. Thus $a=3$ and $b \geq 5$. Moreover, we get $L_{A}=$ $[\mathbf{I}+(b-2) \mathfrak{f}]$, by $(2.5)$. Now let $p: \boldsymbol{F}_{1} \rightarrow \boldsymbol{P}^{1}$ be the ruling projection. Note that $(\mathscr{V} \otimes[-2 \mathfrak{l}])_{\mathfrak{f}}=\mathcal{O}_{\mathfrak{f}} \oplus \mathcal{O}_{\mathfrak{f}}(-1)$ for any fiber $\mathfrak{f}$, since $\mathscr{V}$ is ample and $\operatorname{det} \mathscr{V}=$ $[3 \mathfrak{l}+b \mathfrak{f}]$. So $\mathscr{L}=p_{*}(\mathscr{V} \otimes[-2 \mathfrak{l}])$ is an invertible sheaf on $\boldsymbol{P}^{1}$, and we can put $\mathscr{L}=\mathcal{O}_{\boldsymbol{P}^{1}}(\delta)$ for an integer $\delta$. From the injection $p^{*} \mathscr{L} \rightarrow \mathscr{V} \otimes[-2 \mathrm{l}]$ we obtain an exact sequence

$$
0 \rightarrow[2 \mathfrak{I}+\delta \tilde{\uparrow}] \rightarrow \mathscr{V} \rightarrow \mathscr{Q} \rightarrow 0,
$$

where the quotient is the line bundle $\mathscr{Q}=\operatorname{det} \mathscr{V}-[2 \mathfrak{I}+\delta \mathfrak{\mathfrak { f }}]=[\mathfrak{I}+(b-\delta) \mathfrak{f}]$. Since $\mathscr{V}$ is ample, it follows that $\mathscr{2}$ is ample, hence $b-\delta>1$. Moreover $c_{2}(\mathscr{V})=$ $(2 \mathfrak{l}+\delta \tilde{\mathfrak{f}})(\mathfrak{I}+(b-\delta) \mathfrak{f})=2 b-\delta-2$. Thus the Chern-Wu relation for the tautological line bundle $L$ on $X$ gives

$$
L^{3}=c_{1}(\mathscr{V})^{2}-c_{2}(\mathscr{V})=(3 \mathfrak{I}+b \mathfrak{T})^{2}-(2 b-\delta-2)=4 b+\delta-7 .
$$


On the other hand, since $L_{A}^{2}=(\mathfrak{l}+(b-2) \mathfrak{f})^{2}=2 b-5$ and $L_{B}^{2}=L^{2} \pi^{*} \gamma=$ $\operatorname{deg} \mathscr{V}_{\gamma}=\gamma \operatorname{det} \mathscr{V}=2 b$, we get $L^{3}=L_{A}^{2}+L_{B}^{2}=4 b-5$. Comparing this with (2.7) we obtain $\delta=2$, hence $c_{2}(\mathscr{V})=2 b-4$ and the exact sequence induced by (2.6) on $A$ via the isomorphism $\left.\pi\right|_{A}$ becomes $\left.0 \rightarrow[B]_{A} \rightarrow \pi\right|_{A} ^{*} \mathscr{V} \rightarrow L_{A} \rightarrow 0$. Note that the sequence does not split; otherwise, by restricting (2.6) to I we would get $\mathscr{V}_{\mathrm{I}}=\mathcal{O}_{\mathrm{I}} \oplus \mathcal{O}_{\mathrm{I}}(b-3)$, contradicting the ampleness of $\mathscr{V}$.

The above result is effective as the following example shows.

Example. Let $X$ be the Fano bundle obtained by blowing-up $Y=\boldsymbol{P}\left(T_{\boldsymbol{P}^{2}}\right)$ along a fiber [D, Theorem 3] (see also [SW, case 14 in the Theorem]). Let $\pi: X \rightarrow \boldsymbol{F}_{1}$ be the projection, and let $\beta: \boldsymbol{F}_{1} \rightarrow \boldsymbol{P}^{2}$ be the induced blowing-up of the basis of $Y$. With the same notation as above recall that $I$ is the exceptional divisor of $\beta$ and that $\beta^{*} \mathcal{O}_{\boldsymbol{P}^{2}}(1)=[\mathfrak{I}+\mathfrak{f}]$. Let $\mathscr{V}:=\beta^{*} T_{\boldsymbol{P}^{2}} \otimes[\mathfrak{f}]$. Since the exceptional divisor of the blowing-up $X \rightarrow Y$ is $\pi^{*} \mathrm{I}$, we can write

$$
X=\boldsymbol{P}\left(\beta^{*} T_{\boldsymbol{P}^{2}} \otimes[-\mathfrak{l}]\right)=\boldsymbol{P}(\mathscr{V}) .
$$

Let $L$ be the tautological line bundle of $\mathscr{V}$ on $X$. Then the canonical bundle formula $K_{X}=-2 L+\pi^{*}\left(K_{\boldsymbol{F}_{1}}+\operatorname{det} \mathscr{V}\right)$ gives

$$
2 L=-K_{X}+\pi^{*}(\mathfrak{I}+2 \mathfrak{T}) .
$$

Since $-K_{X}$ is ample and $\pi^{*}(\mathfrak{l}+2 \mathfrak{f})$ is spanned, this shows that $L$ is ample. Moreover $L$ is spanned, $\mathscr{V}$ being so. To see this note that $\beta^{*} T_{\boldsymbol{P}^{2}}$ is very ample on $\boldsymbol{F}_{1} \backslash \mathfrak{I}$ and spanned on $\mathfrak{I}$. Now, consider a smooth curve $\gamma \in|2 \mathfrak{l}+2 \mathfrak{f}|$, and let $B=\pi^{*} \gamma$. Then $B$ is a smooth surface isomorphic to $\boldsymbol{F}_{e}$ for some $e \geq 0$. Note that $L-B$ is the tautological line bundle of

$$
\mathscr{V} \otimes[-\gamma]=\beta^{*} T_{\boldsymbol{P}^{2}} \otimes[-2 \mathrm{l}-\mathfrak{f}]=\beta^{*} T_{\boldsymbol{P}^{2}}(-1) \otimes[-\mathfrak{l}] .
$$

Let $x=\beta(\mathrm{I})$ and let $\mathscr{J}_{x} \subset \mathcal{O}_{\boldsymbol{P}^{2}}$ be the corresponding ideal sheaf. From the Euler sequence we know that $T_{\boldsymbol{P}^{2}}(-1)$ is spanned and $h^{0}\left(T_{\boldsymbol{P}^{2}}(-1)\right)=3$. Then $h^{0}\left(T_{\boldsymbol{P}^{2}}(-1) \otimes \mathscr{J}_{x}\right)=1$. Moreover, since $c_{2}\left(T_{\boldsymbol{P}^{2}}(-1)\right)=1$, the unique surface $S \subset Y$ corresponding to the non-trivial elements in $H^{0}\left(T_{\boldsymbol{P}^{2}}(-1) \otimes \mathscr{J}_{x}\right)$ is a meromorphic section of $Y$ containing exactly one fiber: namely that over $x$. It thus follows that $h^{0}(L-B)=h^{0}\left(\beta^{*} T_{\boldsymbol{P}^{2}}(-1) \otimes[-\mathfrak{I}]\right)=1$. Note that the unique element $A \in|L-B|$ is just the proper transform of $S$ in the blowing-up $X \rightarrow Y$. Therefore $A$ is a smooth surface, which is a meromorphic section of $\pi$. Moreover, the number of fibers of $\pi$ it contains is given by $c_{2}(\mathscr{V} \otimes[-\gamma])$. But it is immediate to check that

$$
c_{2}(\mathscr{V} \otimes[-\gamma])=c_{2}\left(\beta^{*} T_{\boldsymbol{P}^{2}}(-1) \otimes[-\mathfrak{l}]\right)=0 .
$$

Therefore $A$ is a holomorphic section of $\pi$, i.e., $\left.\pi\right|_{A}: A \rightarrow \boldsymbol{F}_{1}$ is an isomorphism.

On the other hand, when $S=\boldsymbol{P}^{2}$, we get the following very precise description of $(X, L)$. 
TheOREM 2.2. Let $X, L, A, B$ be as in (2.0) and assume that $S=\boldsymbol{P}^{2}$. If i) holds, then $(X, L)=\left(\boldsymbol{P}^{1} \times \boldsymbol{P}^{2}, \mathcal{O}_{\boldsymbol{P}^{1} \times \boldsymbol{P}^{2}}(1,2)\right)$, with, up to renaming, $A \in$ $\left|\mathcal{O}_{\boldsymbol{P}^{1} \times \boldsymbol{P}^{2}}(1,0)\right|$ and $B$ a general element of $\left|\mathcal{O}_{\boldsymbol{P}^{1} \times \boldsymbol{P}^{2}}(0,2)\right|$. If ii) holds, then $X=\boldsymbol{P}(\mathscr{V})$, where $\mathscr{V}$ is an ample and spanned vector bundle of rank 2 on $\boldsymbol{P}^{2}$ with Chern classes $c_{1}(\mathscr{V})=4, c_{2}(\mathscr{V})=5$. Moreover, up to renaming, $A \cong \boldsymbol{F}_{1},\left.\pi\right|_{A}$ : $A \rightarrow \boldsymbol{P}^{2}$ being the contraction of the $(-1)$-section of $A$, and $B=\boldsymbol{P}\left(\mathscr{V}_{\gamma}\right) \cong \boldsymbol{P}^{1} \times \boldsymbol{P}^{1}$, $\gamma$ being a smooth conic; furthermore $h=A \cap B$ is a section of $B$ with $h^{2}=0$ and on $A$ it is a smooth element of the linear system $|2 E+2 F|$, where $E$ and $F$ denote the $(-1)$-section and a fiber, respectively.

Proof. Set $X=\boldsymbol{P}(\mathscr{V})$ with $\mathscr{V}:=\pi_{*} L$, as at the beginning of this Section. Since $S=\boldsymbol{P}^{2}$ the situation specializes as follows: $\operatorname{det} \mathscr{V}=\mathcal{O}_{\boldsymbol{P}^{2}}(a)$ with $a \geq 4$ since

$$
H=K_{\boldsymbol{P}^{2}}+\operatorname{det} \mathscr{V}=\mathcal{O}_{\boldsymbol{P}^{2}}(a-3)
$$

is an ample line bundle. Moreover $\gamma \in\left|\mathcal{O}_{P^{2}}(u)\right|$ with $u=1$ or 2 , since $\gamma$ is a smooth rational curve. By (2.2) we know that $H(\operatorname{det} \mathscr{V}-2[\gamma])=0$. So, since $H$ is ample, we get $a=2 u$ and recalling our conditions on $a$ and $u$ we conclude that $4=a=2 u$, i.e., $c_{1}(\mathscr{V})=4$ and $\gamma$ is a smooth conic. In addition, $H=\mathcal{O}_{\boldsymbol{P}^{2}}(1), \pi^{*} H L A=\pi^{*} H L B=2$, by (2.1) and (2.2), and so for the smooth curve $h=A \cap B$ we get

$$
L h=L A B=4 .
$$

Since $B=\pi^{*} \gamma$ we have $B=\boldsymbol{P}\left(\mathscr{V}_{\gamma}\right)$. Thus, in view of the above, we can write $\mathscr{V}_{\gamma}=\mathcal{O}_{\boldsymbol{P}^{1}}\left(e_{1}\right) \oplus \mathcal{O}_{\boldsymbol{P}^{1}}\left(e_{2}\right)$ where $1 \leq e_{1} \leq e_{2}=8-e_{1}$. Hence $\left(B, L_{B}\right)$ is a scroll over $\boldsymbol{P}^{1}$ of invariant $e=8-2 e_{1}$, whose degree is given by

$$
L_{B}^{2}=L^{2} \pi^{*} \gamma=\operatorname{deg} \mathscr{V}_{\gamma}=8 .
$$

Using this information together with (2.8) and the conditions in [Ha, p. 380], a straightforward verification shows that $e=0$, i.e., $B \cong \boldsymbol{P}^{1} \times \boldsymbol{P}^{1}, h$ is an element of the ruling transverse to the projection $\left.\pi\right|_{B}$, and $L_{B}=[h+4 f]$, where $f$ denotes a fiber of $\left.\pi\right|_{B}$. On the other hand, from the Chern-Wu relation for the tautological line bundle $L$ on $X$ we get

$$
L^{3}=c_{1}(\mathscr{V})^{2}-c_{2}(\mathscr{V})=16-c_{2}(\mathscr{V}) .
$$

Note that $\left(\boldsymbol{P}^{2}, \operatorname{det} \mathscr{V}\right)$ is the first reduction of $\left(\Sigma, L_{\Sigma}\right)$, where $\Sigma$ is a general element of $|L|$. Thus $g(X, L)=g\left(\Sigma, L_{\Sigma}\right)=3$. This implies that $c_{2}(\mathscr{V}) \geq 3$, due to results of Lanteri-Sommese [LS] and Noma [N]. Recalling (2.9) and (2.10) we thus see that $\left(A, L_{A}\right)$ has degree

$$
L_{A}^{2}=L^{3}-L_{B}^{2}=8-c_{2}(\mathscr{V}) \leq 5 .
$$

First suppose we are in case i). Then $\left(A, L_{A}\right)=\left(\boldsymbol{P}^{2}, \mathcal{O}_{\boldsymbol{P}^{2}}(v)\right)$, where $v=1$ or 2 in view of (2.11). Moreover, since $h$ corresponds to $\gamma$ in the isomorphism $\left.\pi\right|_{A}: A \rightarrow \boldsymbol{P}^{2}$, we have that $h \in\left|\mathcal{O}_{\boldsymbol{P}^{2}}(2)\right|$; hence

$$
4=L A B=L_{A} h=2 v \text {. }
$$


Thus $v=2, L_{A}^{2}=4$ and so $(2.11)$ gives $c_{2}(\mathscr{V})=4$. On the other hand, since $A$ is a section of $\pi$ there is a surjection from $\mathscr{V}$ to a line bundle on $\boldsymbol{P}^{2}$. This gives rise to an exact sequence

$$
0 \rightarrow \mathcal{O}_{\boldsymbol{P}^{2}}(x) \rightarrow \mathscr{V} \rightarrow \mathcal{O}_{\boldsymbol{P}^{2}}(y) \rightarrow 0,
$$

with $x, y$ integers and $y>0$ since $\mathscr{V}$ is ample. Combining this with the previous conclusions we get

$$
x+y=c_{1}(\mathscr{V})=4=c_{2}(\mathscr{V})=x y,
$$

hence $x=y=2$. On the other hand (2.12) splits, since the first cohomology group of any line bundle on $\boldsymbol{P}^{2}$ is trivial; thus $\mathscr{V}=\left(\mathcal{O}_{\boldsymbol{P}^{2}}(2)\right)^{\oplus 2}$ and this concludes the proof in case i).

Now suppose we are in case ii). Then, checking the list in (1.0) we see that, necessarily, $A=\boldsymbol{F}_{1}$ and $\left.\pi\right|_{A}$ is the contraction of the $(-1)$ section of $A$. Let $E$ and $F$ be the $(-1)$-section and a fiber of $A$, respectively. Since $E$ is a fiber of $\pi$, we have $L_{A} E=L E=1$. Writing $L_{A}=[x E+y F]$ for some integers $x, y$, we thus get $y=x+1$. Then $L_{A}^{2}=x^{2}+2 x$, with $x \geq 1$. Comparing this with (2.11) we see that $x=1$, hence $L_{A}=[E+2 F]$. Note that $E h=0$; otherwise $\gamma=\pi(h)$ would contain the point $p=\pi(E)$, and then $E$ would be in $A \cap B$, contradicting the irreducibility of $h$. On the other hand, $L_{A} h=4$, by (2.8). So, writing $h \sim s E+t F$ for some integers $s, t$, these conditions immediately imply that $t=s=2$, i.e. $h \in|2 E+2 F|$.

To show that the result of Theorem 2.2 is effective, we produce an example as in case ii).

Example. Let $V \subset \boldsymbol{P}^{11}$ be a 4-dimensional classical scroll over $\boldsymbol{P}^{2}$ of degree 11 ; let $\pi: V \rightarrow \boldsymbol{P}^{2}$ be the projection, let $\mathscr{L}$ be the hyperplane line bundle, and let $\mathscr{F}=\pi_{*} \mathscr{L}$ be the corresponding very ample vector bundle of rank 3 . Such a scroll exists, e.g., take $\mathscr{F}=\mathcal{O}_{\boldsymbol{P}^{2}}(2) \oplus \mathcal{O}_{\boldsymbol{P}^{2}}(1)^{\oplus 2}$. Note that "a priori" $(V, \mathscr{L})$ could not be a scroll (in the adjunction theoretic sense). Actually, $K_{V}+3 \mathscr{L}=$ $\pi^{*} H$, where $H=K_{\boldsymbol{P}^{2}}+\operatorname{det} \mathscr{F}=\mathcal{O}_{\boldsymbol{P}^{2}}(a)$ with $a \geq 0$, because $c_{1}(\mathscr{F}) \geq \operatorname{rk} \mathscr{F}=3$, due to the (very) ampleness of $\mathscr{F}$. However, by [F2, Main theorem], the only exception to the ampleness of $K_{\boldsymbol{P}^{2}}+\operatorname{det} \mathscr{F}$ is $\mathscr{\mathscr { F }}=\mathcal{O}_{\boldsymbol{P}^{2}}(1)^{\oplus 3}$. But, recalling the Chern-Wu relation, this would imply that

$$
11=\mathscr{L}^{4}=\mathscr{L}^{2} \pi^{*}\left(c_{1}(\mathscr{F})^{2}-c_{2}(\mathscr{F})\right)=9-3=6,
$$

a contradiction. Therefore the pair $(V, \mathscr{L})$ is a scroll. Now take a general hyperplane section $X \in|\mathscr{L}|$ of $V$ and let $L=\mathscr{L}_{X}$. Then the pair $(X, L)$ is clearly a scroll.

Remarks. a) Note that $B \cong \boldsymbol{P}^{1} \times \boldsymbol{P}^{1}$ in case ii) of Theorem 2.2. However $L_{B}^{2}=8$. So $\left(B, L_{B}\right) \neq\left(\boldsymbol{Q}^{2}, \mathcal{O}_{\boldsymbol{Q}^{2}}(1)\right)$. The same conclusion holds for Theorem 2.1 , since we showed there that $L_{B}^{2}=2 b \geq 10$. 
b) Note also that in case ii) of Theorem 2.2 we have $[A]_{A}=L_{A}-[B]_{A}=$ $[-E]$, hence $A$ is not nef. The same is true for $A$ in Theorem 2.1, since there $[A]_{A}=L_{A}-[B]_{A}=[-\mathfrak{l}+(b-4) \mathfrak{f}]$.

\section{The case when both $\left(A, L_{A}\right),\left(B, L_{B}\right) \in \mathscr{A}$}

Here we start the proof of Theorem (0.1). For the moment we do not need the assumption that $A+B \in|L|$ is a seminef ssd. First we prove some useful lemmas.

LEMmA 3.1. Let $L$ be an ample and spanned line bundle on a smooth projective threefold $X$. Assume that there exists a ssd $A+B \in|L|$, and let $h=A \cap B$ be the corresponding smooth hinge curve. Suppose furthermore that $\left(A, L_{A}\right) \cong\left(\boldsymbol{P}^{2}, \mathcal{O}_{\boldsymbol{P}^{2}}(u)\right)$, with $u=1,2$ and $B \cong \boldsymbol{P}^{2}$ or $\boldsymbol{F}_{e}$ for some $e \geq 0$. Then $g(h)=0$.

Proof. Set $[A]_{A}=\mathcal{O}_{\boldsymbol{P}^{2}}(\delta)$ and $[B]_{A}=\mathcal{O}_{\boldsymbol{P}^{2}}(d)$ for some integers $\delta, d$. Since $\mathcal{O}_{\boldsymbol{P}^{2}}(u)=L_{A}=\mathcal{O}_{\boldsymbol{P}^{2}}(\delta+d)$, we get $d+\delta=u \leq 2$. Note that $h \in\left|B_{A}\right|$. So, assuming, by contradiction, that $g(h)>0$, we get $d \geq 3$, which implies $\delta<0$. So, if $g(h)>0$ we have that $\delta d<0$. On the other hand, looking at the smooth curve $h$ inside $B$, we have

$$
0>\delta d=[A]_{A}[B]_{A}=A^{2} B=[A]_{B}^{2}=h^{2} .
$$

Thus $B \neq \boldsymbol{P}^{2}$. On the other hand, the only irreducible curve on $B \cong \boldsymbol{F}_{e}$ with negative self-intersection is the section $C_{0}$. Therefore $g(h)=g\left(C_{0}\right)=0$, a contradiction.

LEMMA 3.2. Let $L$ be an ample and spanned line bundle on a smooth projective threefold $X$. Assume that there exists a ssd $A+B \in|L|$ and let $h=A \cap B$ be the corresponding smooth hinge curve. Suppose furthermore that $\left(A, L_{A}\right) \cong\left(\boldsymbol{Q}^{2}, \mathcal{O}_{\boldsymbol{Q}^{2}}(1)\right)$ and $B \cong \boldsymbol{F}_{e}$ for some $e \geq 0$. Then $g(h)=0$.

Proof. Set $[B]_{A}=\mathcal{O}_{\boldsymbol{P}^{1} \times \boldsymbol{P}^{1}}(x, y)$ for some integers $x, y$. Then

$$
[A]_{A}=L_{A}-[B]_{A}=\mathcal{O}_{\boldsymbol{P}^{1} \times \boldsymbol{P}^{1}}(1-x, 1-y) .
$$

Since $h \in\left|B_{A}\right|$ is an irreducible curve, we see that $(x, y)$ is either $(1,0),(0,1)$, or $x, y$ are both positive integers. Moreover, if we assume, by contradiction, that $g(h)>0$, then we get $x \geq 2$ and $y \geq 2$; otherwise $h$ would be a section of one of the two rulings of $\boldsymbol{P}^{1} \times \boldsymbol{P}^{1}$. We have

$$
[A]_{A}[B]_{A}=\mathcal{O}_{\boldsymbol{P}^{1} \times \boldsymbol{P}^{1}}(x, y) \mathcal{O}_{\boldsymbol{P}^{1} \times \boldsymbol{P}^{1}}(1-x, 1-y)=x(1-y)+y(1-x)=x+y-2 x y .
$$

Note that the function $\varphi(x, y)=x+y-2 x y$ is always negative for $x \geq 2, y \geq 2$ (actually $\varphi(x, y)=x(1-y)+y(1-x) \leq x(-1)+y(-1) \leq-4)$. On the other hand, looking at the smooth curve $h$ inside $B \cong \boldsymbol{F}_{e}$, we thus see that 


$$
0>\varphi(x, y)=[A]_{A}[B]_{A}=A^{2} B=[A]_{B}^{2}=h^{2} .
$$

But this leads to the same contradiction as in Lemma 3.1.

Now we can proceed with a case-by-case analysis of our ssd $A+B \in|L|$ as in Theorem 0.1 .

(I) Assume that $A \cong \boldsymbol{P}^{2}$ and $B \cong \boldsymbol{P}^{2}$.

Put $[A]_{B}=\mathcal{O}_{P^{2}}\left(d^{\prime}\right)$ and $[B]_{A}=\mathcal{O}_{P^{2}}(d)$ with $d, d^{\prime} \geq 1$.

By Lemma 3.1 we know that $g(h)=0$, hence from the equalities

$$
d(d-3)=\left(K_{A}+[B]_{A}\right)[B]_{A}=2 g(h)-2=\left(K_{B}+[A]_{B}\right)[A]_{B}=d^{\prime}\left(d^{\prime}-3\right)
$$

we get $d^{2}-3 d+2=d^{\prime 2}-3 d^{\prime}+2=0$. Therefore $d, d^{\prime} \in\{1,2\}$. Recall that $L_{A}=\mathcal{O}_{\boldsymbol{P}^{2}}(u)$, with $u=1,2$. Then

$$
d^{\prime 2}=[A]_{B}^{2}=[A]_{A}[B]_{A}=\left(L_{A}-[B]_{A}\right)[B]_{A}=(u-d) d .
$$

This immediately shows that $d^{\prime}=2$ is impossible, while $d^{\prime}=1$ implies $d=1$, $u=2$. Then, letting $L_{B}=\mathcal{O}_{P^{2}}(v)$, from the symmetric relation

$$
1=[B]_{A}^{2}=[A]_{B}[B]_{B}=[A]_{B}\left(L_{B}-[A]_{B}\right)=v-1,
$$

we get $v=2$. So $\left(A, L_{A}\right) \cong\left(B, L_{B}\right) \cong\left(\boldsymbol{P}^{2}, \mathcal{O}_{\boldsymbol{P}^{2}}(2)\right)$.

Moreover $A B L=[B]_{A} L_{A}=d u=2$ and by Lemma 1.1 we obtain that $g(X, L)=1$. So $(X, L)$ is a Del Pezzo threefold and since $L^{3}=L_{A}^{2}+L_{B}^{2}=8$ we see from $[\mathrm{F} 1,(8.5)]$ that $(X, L) \cong\left(\boldsymbol{P}^{3}, \mathcal{O}_{\boldsymbol{P}^{3}}(2)\right)$.

(II) Assume, up to renaming, that $A \cong \boldsymbol{P}^{2}$ and $B \cong Q^{2}$.

Put $[B]_{A}=\mathcal{O}_{\boldsymbol{P}^{2}}(d)$ with $d \geq 1$ and $[A]_{B}=\mathcal{O}_{\boldsymbol{P}^{1} \times \boldsymbol{P}^{1}}(x, y)$ for some nonnegative integers $x$ and $y$. Note that

$$
d^{2}=[B]_{A}^{2}=[B]_{B}[A]_{B}=\left(L_{B}-[A]_{B}\right)[A]_{B}=x+y-2 x y
$$

and

$$
d(d-3)=2 g(h)-2=\left(K_{B}+[A]_{B}\right)[A]_{B}=2 x y-2 x-2 y .
$$

Hence

$$
2 x y-2 x-2 y=d(d-3)=x+y-2 x y-3 d
$$

and this gives

$$
3 x+3 y-4 x y=3 d \geq 3 .
$$

Moreover we have that $A B L=d u=x+y$ for $u=1,2$.

If $u=1$ then $d=x+y$ and from (3.2) we get $x y=0$. Thus we can suppose without loss of generality that $x=0$. Moreover, since $g(h)=0$ by Lemma 3.1, we get $y=1$ and so $d=1$ by (3.2). Then $A B L=x+y=1$ and by Lemma 1.1 we get $g(X, L)=0$. In view of our assumptions it thus follows that $(X, L)$ is a scroll over $\boldsymbol{P}^{1}$ [F1, (12.1) and (5.10)].

If $u=2$ then $2 d=x+y$ and (3.2) gives $3 d=4 x y$. Moreover,

$$
3 x+3 y-4 x y=2 d+d=x+y+d
$$


and then

$$
1 \leq d=2 x+2 y-4 x y .
$$

Since $3 d=4 x y \neq 0$ we get $x \geq 1$ and $y \geq 1$. If $x=1$ then $2-2 y=d \geq 1$ and so $y \leq 1 / 2$, but this is a contradiction. In the same way we see that $y \neq 1$, so we can suppose that $x, y \geq 2$. But then (3.3) would give $1 \leq d<0$, which is absurd.

(III) Finally assume that both $A$ and $B$ are $Q^{2}$.

Put $[A]_{B}=\mathcal{O}_{\boldsymbol{P}^{1} \times \boldsymbol{P}^{1}}(x, y)$ and $[B]_{A}=\mathcal{O}_{\boldsymbol{P}^{1} \times \boldsymbol{P}^{1}}(z, w)$ for some nonnegative integers $x, y, w$ and $z$. Since

and

$$
2 x y=[A]_{B}^{2}=\left(L_{A}-[B]_{A}\right)[B]_{A}=z+w-2 z w
$$

$$
2 z w=[B]_{A}^{2}=\left(L_{B}-[A]_{B}\right)[A]_{B}=x+y-2 x y,
$$

we deduce that

$$
x+y=2(x y+z w)=z+w .
$$

By Lemma 3.2, the genus formula for $h \in\left|A_{B}\right|$ gives

$$
-2=2 g(h)-2=\left(K_{B}+[A]_{B}\right)[A]_{B}=2 x y-2 x-2 y,
$$

hence $(x-1)(y-1)=0$. Similarly, arguing on $h \in\left|B_{A}\right|$ we get $(z-1)(w-1)=$ 0 . Up to exchanging the factors of $A$ and $B$ we can thus assume that $x=z=1$. But then (3.4) gives $y=w$ and $1+y=2(y+w)=4 y$, which is impossible, $y$ being an integer.

We sum up the above results in the following statement

THEOREM 3.3. Let $L$ be an ample and spanned line bundle on a smooth projective threefold $X$. Assume that there is a ssd $A+B \in|L|$. If both $\left(A, L_{A}\right)$ and $\left(B, L_{B}\right)$ are in class $\mathscr{A}$ then $(X, L)$ is either a scroll over $\boldsymbol{P}^{1}$ or $\left(\boldsymbol{P}^{3}, \mathcal{O}_{\boldsymbol{P}^{3}}(2)\right)$.

Remark. Note that, at least in cases (I) and (II), we could deduce the result above also from [CHS, (3.10)], in view of Lemma 3.1, after a case-by-case analysis of the polarized threefolds appearing there (e.g., see the argument at the end of Section 5). However we preferred to provide a more direct proof.

\section{The case when $\left(A, L_{A}\right) \in \mathscr{A}$ and $\left(B, L_{B}\right) \in \mathscr{B}$}

We proceed with a case-by-case analysis also in this context, continuing the enumeration started in Section 3 .

(IV) Assume that $\left(A, L_{A}\right) \cong\left(\boldsymbol{P}^{2}, \mathcal{O}_{\boldsymbol{P}^{2}}(u)\right)$ with $u=1,2$.

Put $[B]_{A}=\mathcal{O}_{\boldsymbol{P}^{2}}(d)$ with $d \geq 1$. Let $B \cong \boldsymbol{F}_{e}$ for some $e \geq 0$ and let $C_{0}$ and $f$ be a section of minimal self-intersection and a fiber respectively. Since $\left(B, L_{B}\right)$ is a scroll we can write $L_{B}=\left[C_{0}+\beta f\right]$ for some integer $\beta>e$. Note that $h \in\left|A_{B}\right|$ is a smooth curve of genus zero on $\boldsymbol{F}_{e}$ by Lemma 3.1. Hence by [Ha, p. 380] there are three possibilities: 
(1) $[A]_{B}=[f]$;

(2) $[A]_{B}=\left[C_{0}\right]$;

(3) $[A]_{B}=\left[x C_{0}+y f\right]$ with $x>0$ and $y>x e$, or $e>0, x>0$ and $y=x e$.

CASE (1). We observe that $[A]_{B} L_{B}=A B L=1$ and then $g(X, L)=0$ by Lemma 1.1. After ruling out $\left(\boldsymbol{P}^{3}, \mathcal{O}_{\boldsymbol{P}^{3}}(1)\right)$ and $\left(\boldsymbol{Q}^{3}, \mathcal{O}_{\boldsymbol{Q}^{3}}(1)\right)$, we deduce that $(X, L)$ is a scroll over $\boldsymbol{P}^{1}\left[\mathrm{~F} 1,(12.1)\right.$ and (5.10)], with $\left(A, L_{A}\right) \cong\left(\boldsymbol{P}^{2}, \mathcal{O}_{\boldsymbol{P}^{2}}(1)\right)$.

CASE (2). Since $g(h)=0$, by the genus formula we obtain

$$
-2=2 g(h)-2=\left(K_{A}+[B]_{A}\right)[B]_{A}=(d-3) d,
$$

i.e., $d^{2}-3 d+2=0$. Thus $d=1$ or 2 .

Let $d=1$. Thus $[A]_{A}=L_{A}-[B]_{A}=\mathcal{O}_{P^{2}}(u-1)$ with $u=1,2$. If $u=2$ then we get $[A]_{A}=\mathcal{O}_{P^{2}}(1)$ and so

$$
1=[A]_{A}[B]_{A}=[A]_{B}^{2}=C_{0}^{2}=-e \leq 0,
$$

but this is a contradiction. If $u=1$ then $[A]_{A}=\mathcal{O}_{\boldsymbol{P}^{2}}$ and $[B]_{A} L_{A}=A B L=1$. So we get $g(X, L)=0$ by Lemma 1.1, and again $(X, L)$ is a scroll over $\boldsymbol{P}^{1}$.

Now let $d=2$. Thus $[A]_{A}=\mathcal{O}_{P^{2}}(u-2)$ with $u=1,2$. If $u=2$ then $0=$ $[A]_{A}[B]_{A}=[A]_{B}^{2}=-e$. Moreover $\quad 4=[B]_{A} L_{A}=A B L=[A]_{B} L_{B}=C_{0}\left(C_{0}+\beta f\right)$, which gives $L_{B}=\left[C_{0}+4 f\right]$. Therefore

$$
\left[K_{X}+2 L\right]_{B} f=\left(K_{B}+[A]_{B}+L_{B}\right) f=2 f^{2}=0 .
$$

This shows that $K_{X}+2 L$ cannot be ample. Note that $(X, L)$ can be neither $\left(\boldsymbol{P}^{3}, \mathcal{O}_{\boldsymbol{P}^{3}}(1)\right)$ nor $\left(\boldsymbol{Q}^{3}, \mathcal{O}_{\boldsymbol{Q}^{3}}(1)\right)$. Hence, from adjunction theory we know that $(X, L)$ belongs to a very short list of pairs. If $K_{X}+2 L$ is not nef, then by [CHS, (3.1)] $(X, L)$ is

(A) a scroll over a smooth curve,

but this is absurd, because $L_{A}=\mathcal{O}_{P^{2}}(2)$, and this polarization on $A$ is not compatible with Lemma 1.2. Now suppose that $K_{X}+2 L$ is nef but not big. Then, by [CHS, (3.2)], $(X, L)$ is one of the following pairs:

(B) a Del Pezzo threefold;

(C) a quadric fibration over a smooth curve;

(D) a scroll over a smooth surface.

Since

$$
[B]_{A} L_{A}=A B L=4,
$$

Lemma 1.1 shows that $g(X, L)=3$. Clearly this rules out case (B).

Case (C) cannot occur as well. Actually, let $q: X \rightarrow C$ be the quadric fibration over a smooth curve $C$. Since $A \cong \boldsymbol{P}^{2}, A$ cannot be a fiber of $q$ and so $\left.q\right|_{A}: A \rightarrow C$ is a surjection. But this is impossible since $\boldsymbol{P}^{2}$ cannot fibre over a curve.

In case (D) we conclude that $(X, L)$ is a scroll over $\boldsymbol{P}^{2}$ as in Theorem 2.2, case i), because $A \cong \boldsymbol{P}^{2}$.

Assume now that $K_{X}+2 L$ is nef and big. Then by [CHS, (3.5)] 
(E) there exists the first reduction morphism $\rho:(X, L) \rightarrow\left(X^{\prime}, L^{\prime}\right)$ and the adjoint bundle $K_{X^{\prime}}+2 L^{\prime}$ is ample.

Since $K_{X}+2 L$ is not ample, clearly $\rho$ is not an isomorphism. So, by [CHS, (3.5), (3.6)] we get the following possibilities:

$\left(\mathrm{E}_{1}\right)$ one of the two components of $A+B \in|L|$ is an exceptional divisor of $\rho$, or

$\left(\mathrm{E}_{2}\right) \rho$ contracts at least one exceptional divisor $E$ and neither $A$ nor $B$ is one of them.

But case $(\mathrm{E})$ cannot occur. Indeed, in case $\left(\mathrm{E}_{1}\right)$ we have that $A=E$ is an exceptional divisor of $\rho$; then $A B L=[B]_{E} L_{E}=\mathcal{O}_{\boldsymbol{P}^{2}}(2) \mathcal{O}_{\boldsymbol{P}^{2}}(1)=2$, which contradicts (4.1). On the other hand, in case $\left(\mathrm{E}_{2}\right)$ clearly $A \cap E=\emptyset$ while $B \cap E$ is a rational curve on $B$, which is contracted by $\rho$. But this is impossible, since, as we have seen $B \cong \boldsymbol{F}_{0}$.

Finally suppose that $u=1$. Then $[A]_{A}=\mathcal{O}_{\boldsymbol{P}^{2}}(-1),[B]_{A}=\mathcal{O}_{\boldsymbol{P}^{2}}(2)$ and so we get $-2=[A]_{A}[B]_{A}=[A]_{B}^{2}=-e$. Since

$$
2=A B L=[A]_{B} L_{B}=C_{0}\left[C_{0}+\beta f\right]=-e+\beta=-2+\beta,
$$

we conclude that $L^{3}=L_{A}^{2}+L_{B}^{2}=1+\left[C_{0}+4 f\right]^{2}=7$ and by Lemma 1.1 we obtain that $g(X, L)=1$, i.e., $(X, L)$ is a Del Pezzo threefold of degree 7 . Therefore $X$ is $\boldsymbol{P}^{3}$ blown-up at a point $p$ and $L=\rho^{*} \mathcal{O}_{\boldsymbol{P}^{3}}(2)-E$, where $\rho: X \rightarrow \boldsymbol{P}^{3}$ is the blowing-up and $E=\rho^{-1}(p)$ is the exceptional divisor [F1, $(8.6)]$.

CASE (3). Since

$$
(u-d) d=[A]_{A}[B]_{A}=[A]_{B}^{2}=\left[x C_{0}+y f\right]^{2}=x(2 y-e x)>0
$$

and $1 \leq d, u \leq 2$, we deduce that $d=1, u=2$ and $x=1$. This gives $A B L=$ $[A]_{B}^{2}+[B]_{A}^{2}=2$ and by Lemma 1.1 we have that $g(X, L)=1$, i.e., $(X, L)$ is a Del Pezzo threefold.

Furthermore, from $x=1$ we get $[B]_{B}^{2}=\left(L_{B}-[A]_{B}\right)^{2}=(\beta-y)^{2} f^{2}=0$. So it follows that $L_{B}^{2}=\left(A^{2}+2 A B+B^{2}\right) B=[A]_{A}[B]_{A}+2[B]_{A}^{2}=3$ and then $L^{3}=$ $L_{A}^{2}+L_{B}^{2}=4+3=7$. This leads to the same conclusion as at the end of case (2).

The discussion above proves the following

THEOREM 4.1. Let $L$ be an ample and spanned line bundle on a smooth projective threefold $X$. Assume that there is a ssd $A+B \in|L|$. If $\left(A, L_{A}\right) \cong$ $\left(\boldsymbol{P}^{2}, \mathcal{O}_{\boldsymbol{P}^{2}}(u)\right)$ with $u=1,2$ and $\left(B, L_{B}\right) \in \mathscr{B}$ then $(X, L)$ is one of the following pairs:

1. a scroll over $\boldsymbol{P}^{1}$;

2. $\left(\boldsymbol{P}^{1} \times \boldsymbol{P}^{2}, \mathcal{O}_{\boldsymbol{P}^{1} \times \boldsymbol{P}^{2}}(1,2)\right)$, with $A \in\left|\mathcal{O}_{\boldsymbol{P}^{1} \times \boldsymbol{P}^{2}}(1,0)\right|$ and $B \in\left|\mathcal{O}_{\boldsymbol{P}^{1} \times \boldsymbol{P}^{2}}(0,2)\right| a$ general element;

3. $(X, L)$ has $\left(\boldsymbol{P}^{3}, \mathcal{O}_{\boldsymbol{P}^{3}}(2)\right)$ as first reduction with $X$ being $\boldsymbol{P}^{3}$ blown-up at one point. 
(V) Suppose now that $\left(A, L_{A}\right) \cong\left(\boldsymbol{Q}^{2}, \mathcal{O}_{\mathbf{Q}^{2}}(1)\right)$.

By the genus formula and Lemma 3.2 we get

$$
-2=2 g(h)-2=\left(K_{A}+[B]_{A}\right)[B]_{A}=\left(-L_{A}+[A]_{A}\right)[B]_{A}=-A B L-[A]_{B}^{2} .
$$

We claim that $[A]_{B}^{2} \leq 0$. If not, then the equation above gives $A B L<$ $A B L+[A]_{B}^{2}=2$, and then Lemma 1.1 would imply $g(X, L)=0$. But, clearly, $(X, L)$ can be neither $\left(\boldsymbol{P}^{3}, \mathcal{O}_{\boldsymbol{P}^{3}}(1)\right)$, nor $\left(\boldsymbol{Q}^{3}, \mathcal{O}_{\boldsymbol{O}^{3}}(1)\right)$, because $\left(B, L_{B}\right) \in \mathscr{B}$. Moreover $(X, L)$ cannot be a scroll over a smooth curve, since our ssd $A+B$ is not compatible with Lemma 1.2. This proves the claim. Note that it cannot be $[A]_{B}=[f]$. Otherwise we would get

$$
2=A B L=[B]_{A}^{2}=[B]_{B}[A]_{B}=[B]_{B} f=\left(L_{B}-[A]_{B}\right) f=L_{B} f=1,
$$

a contradiction. If $[A]_{B} \neq\left[C_{0}\right]$, recalling that $h \in\left|A_{B}\right|$ is a smooth curve, we can write $[A]_{B}=\left[a C_{0}+b f\right]$, where $a>0, b>a e$ or $e>0, a>0$ and $b=a e$ [Ha, p. 380]. But in these cases we have $[A]_{B}^{2}=a(2 b-a e)>0$, which contradicts the claim. All this shows that $[A]_{B}=\left[C_{0}\right]$.

On the other hand, since $A \cong \boldsymbol{Q}^{2}=\boldsymbol{P}^{1} \times \boldsymbol{P}^{1}$, we can write $[B]_{A}=$ $\mathcal{O}_{\boldsymbol{P}^{1} \times \boldsymbol{P}^{1}}(x, y)$ for some integers $x, y \geq 0$. Recall that $h \in\left|B_{A}\right|$ has genus zero by Lemma 3.2. So, up to exchanging the factors of $A$, we can suppose that $x=1$. Then we get

$$
-e=[A]_{B}^{2}=[A]_{A}[B]_{A}=L_{A}[B]_{A}-[B]_{A}^{2}=1-y .
$$

Therefore $[B]_{A}=\mathcal{O}_{\boldsymbol{P}^{1} \times \boldsymbol{P}^{1}}(1, e+1)$ and $[A]_{A}=\mathcal{O}_{\boldsymbol{P}^{1} \times \boldsymbol{P}^{1}}(0,-e)$. By adjunction we thus get

$$
\left[K_{X}+2 L\right]_{A}=K_{A}+L_{A}+[B]_{A}=\mathcal{O}_{\boldsymbol{P}^{1} \times \boldsymbol{P}^{1}}(0, e)
$$

and this shows that $K_{X}+2 L$ is not ample. Arguing as in (IV) and noting that neither $A$ nor $B$ can be an exceptional divisor of the first reduction morphism, we conclude that $(X, L)$ is one of the following pairs:

(A) a scroll over a smooth curve;

(B) a Del Pezzo threefold;

(C) a quadric fibration over a smooth curve;

(D) a scroll over a smooth surface.

$\left(\mathrm{E}_{2}\right)$ there exists the first reduction morphism $\rho:(X, L) \rightarrow\left(X^{\prime}, L^{\prime}\right)$ and the adjoint bundle $K_{X^{\prime}}+2 L^{\prime}$ is ample; $\rho$ contracts at least one exceptional divisor $E$ and neither $A$ nor $B$ is one of them.

Case (A) cannot occur since the structure of $A$ and $B$ is not compatible with Lemma 1.2 .

To deal with case $(\mathrm{B})$, let $L_{B}=\left[C_{0}+\beta f\right]$ with $\beta>e$. Since $g(h)=0$, we have that

$$
\beta-e=C_{0}\left(C_{0}+\beta f\right)=[A]_{B} L_{B}=2-[A]_{B}^{2}=e+2,
$$

i.e. $\beta=2 e+2$. By Lemma 1.1 we know that $A B L=g(X, L)+1=2$. Then $e=0, \beta=2$, i.e. $\left(B, L_{B}\right)=\left(\boldsymbol{F}_{0},\left[C_{0}+2 f\right]\right)$. Therefore $L^{3}=L_{A}^{2}+L_{B}^{2}=2+L_{B}^{2}=6$ and by $[\mathrm{F} 1,(8.7)]$ we get $(X, L)=\left(\boldsymbol{P}^{1} \times \boldsymbol{P}^{1} \times \boldsymbol{P}^{1}, \mathcal{O}_{\boldsymbol{P}^{1} \times \boldsymbol{P}^{1} \times \boldsymbol{P}^{1}}(1,1,1)\right)$. 
In case (C), let $q: X \rightarrow C$ be the quadric fibration morphism. Then by Lemma 1.4 we deduce that $C \cong \boldsymbol{P}^{1}$.

Case (D) does not occur since the present polarization of $A$ is not compatible with that of the component $\boldsymbol{P}^{1} \times \boldsymbol{P}^{1}$ arising in the proofs of Theorems 2.1 and 2.2 (see Remark a) at the end of Section 2).

Finally, case $\left(\mathrm{E}_{2}\right)$ is not possible. Indeed, since $L_{E}=[A]_{E}+[B]_{E}=\mathcal{O}_{\boldsymbol{P}^{2}}(1)$ and $A \cong \boldsymbol{P}^{1} \times \boldsymbol{P}^{1}$, we deduce that $[A]_{E}=\mathcal{O}_{\boldsymbol{P}^{2}}$ and $[B]_{E}=\mathcal{O}_{\boldsymbol{P}^{2}}(1)$. Thus $E_{B} \subset B$ is a $(-1)$-curve and so this gives $E_{B}=C_{0}, B \cong \boldsymbol{F}_{1}$ and by arguing as in case (B), we see that $L_{B}=\left[C_{0}+4 f\right]$. Thus we get

$$
3=C_{0}\left(C_{0}+4 f\right)=E_{B} L_{B}=E B^{2}+E B A=B_{E}^{2}=1,
$$

but this is absurd.

We can sum up the above results in the following

THEOREM 4.2. Let $L$ be an ample and spanned line bundle on an irreducible projective manifold $X$ of dimension three. Assume that there is a ssd $A+B \in|L|$. If $\left(A, L_{A}\right) \cong\left(\boldsymbol{Q}^{2}, \mathcal{O}_{Q^{2}}(1)\right)$ and $\left(B, L_{B}\right) \in \mathscr{B}$ then $(X, L)$ is one of the following pairs:

1. a quadric fibration over $\boldsymbol{P}^{1}$; or

2. $\left(\boldsymbol{P}^{1} \times \boldsymbol{P}^{1} \times \boldsymbol{P}^{1}, \mathcal{O}_{\boldsymbol{P}^{1} \times \boldsymbol{P}^{1} \times \boldsymbol{P}^{1}}(1,1,1)\right)$.

\section{The case when both $\left(A, L_{A}\right),\left(B, L_{B}\right) \in \mathscr{B}$}

When both components of the ssd $A+B \in|L|$ are rational scrolls we need a further condition to control the genus of the smooth curve $h=A \cap B$. This is provided by the notion of seminef decomposition which we recall for the convenience of the reader (see $[\mathrm{T}, \S 1]$ ).

Definition. Let $(X, L)$ be a polarized manifold of dimension $n \geq 3$ and let $A+B \in|L|$ be a ssd. We say that $A+B$ is a seminef divisor or shortly a seminef ssd, if at least one of $A$ and $B$ is a nef divisor or at worst an exceptional divisor of the first reduction mapping of $(X, L)$.

Remark. Note that all ssd of sectional genera zero allowed for the pairs $(X, L)$ listed in Theorems 2.2, 3.3, 4.1 and 4.2 are seminef. So these results remain unchanged under the extra assumption that $A+B \in|L|$ is a seminef ssd. In particular this gives cases $1,2,5,6$ and 8 in Theorem 0.1 .

The next lemma shows the role of the seminefness assumption.

Lemma 5.1. Let $L$ be an ample and spanned line bundle on a smooth projective threefold $X$ and let $A+B \in|L|$ be a seminef ssd. If both $\left(A, L_{A}\right)$, $\left(B, L_{B}\right) \in \mathscr{B}$, then the corresponding hinge curve $h=A \cap B$ has genus $g(h)=0$.

Note that in the present setting, neither $A$ nor $B$ can be an exceptional divisor of the first reduction morphism. Hence, due to the assumption, one of 
them, say $A$, is nef. Then the assertion above is an obvious corollary of the following more general result.

LEMMA 5.2. Let $L$ be simply an ample line bundle on a smooth projective threefold $X$, and suppose that $A+B \in|L|$ is a ssd. If $A$ is a nef divisor and $\left(A, L_{A}\right)$ is a scroll over a smooth curve of genus $p$, then the hinge curve $h$ has genus $g(h)=0$ or $p$.

Proof. Let $C_{0}$ and $f$ be a section of minimal self-intersection and a fiber of $A$ respectively. Since $h \in\left|B_{A}\right|$, we can write $[B]_{A} \equiv\left[x C_{0}+y f\right]$ (numerical equivalence) for some integers $x, y$, where $x=h f \geq 0$. On the other hand, since $\left(A, L_{A}\right)$ is a scroll, we have that $L_{A} \equiv\left[C_{0}+t f\right]$ for a suitable integer $t>0$. Thus

$$
[A]_{A}=L_{A}-[B]_{A} \equiv\left[(1-x) C_{0}+(t-y) f\right],
$$

where $1-x=A f \geq 0$, due to the nefness of $A$. Now, if $g(h) \leq p$, it can only be $g(h)=0$ or $p$, by the Riemann-Hurwitz theorem. So, let $g(h)>p$. Then $x \geq 2$, otherwise $h$ would be either a fiber (if $x=0$ ) or a section of $A$ (if $x=1$ ), which implies $g(h)=0$ or $p$, respectively. But this clearly contradicts the inequality $x \leq 1$ obtained before.

The fact that $g(h)=0$ under the assumption that $A+B \in|L|$ is a seminef ssd with $g\left(A, L_{A}\right)=g\left(B, L_{B}\right)=0$ also follows from [T, Lemma 6]. However, we preferred to present here a direct proof of this fact in line with that of Lemmas 3.1 and 3.2.

To conclude the proof of Theorem 0.1 , consider our seminef ssd $A+B \in|L|$. If $K_{X}+2 L$ is not nef and big, we know that $(X, L)$ belongs to a very precise list of pairs [S]. On the other hand, if $K_{X}+2 L$ is nef and big, let $\rho: X \rightarrow X^{\prime}$ be the reduction morphism. Since $\left(A, L_{A}\right)$ and $\left(B, L_{B}\right)$ are in $\mathscr{B}, \rho$ can contract neither $A$ nor $B$. So, one of them, say $A$, is a nef divisor and then we deduce from Lemma 5.1 that $g(h)=0$. Then [CHS, (3.10)] applies. Note that case 5 in [CHS, (3.10)] cannot occur. Thus we obtain that $(X, L)$ is one of the following threefolds:

(A) a scroll over a smooth curve;

(B) a Del Pezzo threefold;

(C) a quadric fibration over a smooth curve;

(D) a scroll over a smooth surface;

(E) $K_{X}+2 L$ is nef and big and there exists the first reduction morphism $\rho:(X, L) \rightarrow\left(X^{\prime}, L^{\prime}\right)$; moreover, the adjoint bundle $K_{X^{\prime}}+2 L^{\prime}$ is ample and neither $A$ nor $B$ is a fiber of $\rho$. Let $A^{\prime}=\rho(A)$ and $B^{\prime}=\rho(B)$. The following cases can occur:

(E1) $\left(X^{\prime}, L^{\prime}\right) \cong\left(\boldsymbol{Q}^{3}, \mathcal{O}_{\boldsymbol{Q}^{3}}(2)\right)$.

(E2) $\left(X^{\prime}, L^{\prime}\right) \cong\left(\boldsymbol{P}^{3}, \mathcal{O}_{\boldsymbol{P}^{3}}(3)\right)$;

(E3) $X^{\prime}$ is a $\boldsymbol{P}^{2}$-bundle over a smooth curve $Y, \quad v: X^{\prime} \rightarrow Y$ with $2 K_{X^{\prime}}+3 L^{\prime}=v^{*} H$ for an ample line bundle $H$ on $Y$. Thus $\left(F, L_{F}^{\prime}\right)=$ 
$\left(\boldsymbol{P}^{2}, \mathcal{O}_{\boldsymbol{P}^{2}}(2)\right)$ for a general fiber $F$. In the present setting there are two possibilities:

(E3-i) after renaming, if necessary, $A^{\prime}$ is a fiber of $v, Y=\boldsymbol{P}^{1}$, and $B^{\prime}$ meets a general fiber in a smooth conic; or

(E3-ii) both $A^{\prime}$ and $B^{\prime}$ are $\boldsymbol{P}^{1}$-bundles over $Y=\boldsymbol{P}^{1}$ via $v$, each meeting a fiber of $v$ in a line.

Since $\left(A, L_{A}\right)$ and $\left(B, L_{B}\right)$ are in $\mathscr{B}$, case $(\mathrm{A})$ is not possible.

Case (B) occurs: in fact, by combining [CHS, (3.4)] with Lemma 1.3 and the subsequent remark we see that $(X, L)=\left(\boldsymbol{P}\left(T_{\boldsymbol{P}^{2}}\right), \xi_{T_{\boldsymbol{P}^{2}}}\right)$.

Case $(\mathrm{C})$ can also occur, and the base curve is $\boldsymbol{P}^{1}$ by Lemma 1.4, since both $A$ and $B$ are $\boldsymbol{P}^{1}$-bundles over $\boldsymbol{P}^{1}$.

Case (D) leads to the pairs in Theorem 2.1 and in case ii) of Theorem 2.2. We observe that cases (E1) and (E2) cannot occur since $g\left(A, L_{A}\right)=g\left(B, L_{B}\right)=0$.

Case (E3-i) is not possible. Since $L_{A^{\prime}}^{\prime}=L_{F}^{\prime}=\mathcal{O}_{P^{2}}(2)$ we have

$$
A^{\prime} B^{\prime} L^{\prime}=\left[B^{\prime}\right]_{F} L_{F}^{\prime}=\left(L_{F}^{\prime}\right)^{2}=4
$$

and so by Lemma 1.1 we obtain that $g\left(X^{\prime}, L^{\prime}\right)=3$. Moreover $2 K_{X^{\prime}}+3 L^{\prime}=$ $v^{*} H \equiv t F$ for some integer $t \geq 1$ and then by the genus formula we get

$$
\begin{aligned}
8 & =2\left(2 g\left(X^{\prime}, L^{\prime}\right)-2\right)=2\left(K_{X^{\prime}}+2 L^{\prime}\right)\left(L^{\prime}\right)^{2} \\
& =\left(2 K_{X^{\prime}}+3 L^{\prime}+L^{\prime}\right)\left(L^{\prime}\right)^{2}=t\left(L_{F}^{\prime}\right)^{2}+\left(L^{\prime}\right)^{3} \\
& =4 t+\left(L^{\prime}\right)^{3} \geq 4 t+1 .
\end{aligned}
$$

Thus $t=1$ and $\left(L^{\prime}\right)^{3}=4$. But this gives $4=\left(L^{\prime}\right)^{3}=\left(L_{F}^{\prime}\right)^{2}+\left(L_{B^{\prime}}^{\prime}\right)^{2}=$ $4+\left(L_{B^{\prime}}^{\prime}\right)^{2}$ which is absurd since $L^{\prime}$ is ample.

Finally, also case (E3-ii) is not possible. Since $p=\left.v\right|_{A^{\prime}}: A^{\prime} \rightarrow \boldsymbol{P}^{1}$ is a $\boldsymbol{P}^{1}$. bundle and $\left(L_{A^{\prime}}^{\prime}\right)_{f}=\mathcal{O}_{P^{1}}(1)$ for every fiber $f$ of $p$, we know that $K_{A^{\prime}}+2 L_{A^{\prime}}^{\prime}=$ $p^{*} H^{\prime} \equiv t f$ for some integer $t$. As observed before, we can assume without loss of generality that $A$ is nef, hence $A^{\prime}$ is nef. Therefore

$$
\left(K_{X^{\prime}}+2 L^{\prime}\right)_{A^{\prime}} f=\left(K_{A^{\prime}}+L_{A^{\prime}}^{\prime}+\left[B^{\prime}\right]_{A^{\prime}}\right) f \leq\left(K_{A^{\prime}}+2 L_{A^{\prime}}^{\prime}\right) f=t[f]^{2}=0,
$$

but this is a contradiction since we know that $K_{X^{\prime}}+2 L^{\prime}$ is ample.

Summing up the discussion above we get cases 2 again, 3, 4 and 7 of Theorem 0.1. Combining this with the remark before Lemma 5.1 concludes the proof of Theorem 0.1 .

\section{REFERENCES}

[BS] M. C. Beltrametti, A. J. Sommese, The Adjunction Theory of Complex Projective Varieties, Expositions in Mathematics, vol. 16, W. de Gruyter, Berlin-New York, 1995.

[BCS] M. C. Beltrametti, K. A. Chandler, and A. J. Sommese, Reducible hyperplane sections, II, Kodai Math. J. 25 (2002), no. 2, 139-150.

[CHS $]$ K. A. Chandler, A. Howard and A. J. Sommese, Reducible hyperplane sections I, J. Math. Soc. Japan 51 (1999), 887-910. 
[D] I. V. DËMIN, Three dimensional Fano manifolds representable in the form of line bundles (Russian), Izv. Acad. Nauk SSSR, 44 (1980); English translation in Math. USSR Ivz. 17 (1981), 219-226. Addendum to this paper in Izv. Acad. Nauk SSSR, 46 (1982); English translation in Math. USSR Ivz. 20 (1983), 625-626.

[F1] T. Fusita, Classification Theories of Polarized Varieties, London Math. Soc. Lecture Notes Series, vol. 155, Cambridge Univ. Press, Cambridge, 1990.

[F2] T. Fustra, On adjoint bundles of ample vector bundles, in Complex Algebraic Varieties, Bayreuth 1990, Lecture Notes in Math. 1507 (1992), 105-112, Springer-Verlag, New York.

[Ha] R. Hartshorne, Algebraic Geometry, Springer-Verlag, New York, 1978.

[LS] A. Lanteri, A. J. Sommese, A vector bundle characterization of $\boldsymbol{P}^{n}$, Abh. Math. Sem. Univ. Hamburg 58 (1988), 89-96.

[LT] A. Lanteri, A. L. Tironi, On reducible hyperplane sections of 4-folds, J. Math. Soc. Japan 53 (2001), 559-563.

[N] A. Noma, Classification of rank-2 ample and spanned vector bundles on surfaces whose zero loci consist of general points, Trans. Amer. Math. Soc. 342 (1994), 867-894.

[S] A. J. Sommese, On the adjunction theoretic structure of projective varieties, in Proceedings of the Complex Analysis and Algebraic Geometry Conference, Göttingen 1985, Lecture Notes in Math. 1194 (1986), 175-213, Springer-Verlag, New York.

[SW] M. SzureK, J. A. Wiśniewski, Fano bundles of rank 2 on surfaces, Compositio Math. 76 (1990), 295-305.

[T] A. L. Tironi, Reducible hyperplane sections of 4-folds: seminef decompositions with low sectional genera, Istituto Lombardo (Rend. Sc.), A 134 (2000), 47-58.

AUTHORS' ADDRESS:

Dipartimento di Matematica "F. EnRiques"-Università

Via C. Saldini, 50

I-20133 Milano, Italy

lanteri@mat.unimi.it

atironi@mat.unimi.it 